\title{
NUMERICAL STUDY ON SIGNIFICANCE OF WIND ACTION ON 2-D FREAK WAVES WITH DIFFERENT PARAMETERS
}

Shiqiang Yan

School of Engineering and Mathematical Sciences, City University, London, UK.

Qingwei Ma

School of Engineering and Mathematical Sciences, City University, London, UK., q.ma@city.ac.uk

Follow this and additional works at: https://jmstt.ntou.edu.tw/journal

Part of the Mechanical Engineering Commons

\section{Recommended Citation}

Yan, Shiqiang and Ma, Qingwei (2012) "NUMERICAL STUDY ON SIGNIFICANCE OF WIND ACTION ON 2-D FREAK WAVES WITH DIFFERENT PARAMETERS," Journal of Marine Science and Technology. Vol. 20: Iss. 1, Article 2. DOI: $10.51400 / 2709-6998.2417$

Available at: https://jmstt.ntou.edu.tw/journal/vol20/iss1/2

This Research Article is brought to you for free and open access by Journal of Marine Science and Technology. It has been accepted for inclusion in Journal of Marine Science and Technology by an authorized editor of Journal of Marine Science and Technology. 
NUMERICAL STUDY ON SIGNIFICANCE OF WIND ACTION ON 2-D FREAK WAVES WITH DIFFERENT PARAMETERS

Acknowledgements

This work is sponsored by Leverhulme Trust, UK, for which the authors are most grateful. 


\title{
NUMERICAL STUDY ON SIGNIFICANCE OF WIND ACTION ON 2-D FREAK WAVES WITH DIFFERENT PARAMETERS
}

\author{
Shiqiang Yan and Qingwei Ma
}

Key words: wind effects, freak waves, air pressure, QALE-FEM, numerical simulation.

\begin{abstract}
This paper presents a numerical study on how the significance of wind action differs when varying the wave parameters. The quasi arbitrary Lagrangian-Eulerian finite element method (QALE-FEM) is used for this purpose. An improved model for wind-excited pressure and wind-driven current, which is recently developed by the authors of this paper [27], is coupled with the QALE-FEM. Many cases involving freak waves with different focusing time/point and frequency ranges under the action of winds are investigated. The results show that the significance of wind actions on freak waves strongly depends on the focusing time, the focusing point and the frequency range. The knowledge does not only help the proper set up of experiments studying wind effects on freak waves but also contributes to the development of a method for predicting freak waves.
\end{abstract}

\section{INTRODUCTION}

Freak waves are extreme wave events occurring in a small region during a short time. Such events have been observed not only in deep water, but also in shallow water. For example, the famous New Year wave recorded at the Draupner platform in the North Sea on 1st, January, 1995 [3] showed a freak wave with characteristic wavelength of $220 \mathrm{~m}$ occurs in an area of $70 \mathrm{~m}$ water depth. Freak waves may pose a real threat to human activities in the oceans [8]. To prevent damages caused by a freak wave from happening, it is essential to accurately predict the position (focusing point) and the time (focusing time) it happens, as well as the maximum wave height it may cause. Many efforts have been devoted for this purpose. The corresponding reviews can be found in $[7,9,25]$. Neverthe-

Paper submitted 04/26/10; Revised 06/05/10; accepted 06/10/2010. Author for correspondence: Qingwei Ma (e-mail: q.ma@city.ac.uk).

School of Engineering and Mathematical Sciences, City University, London, $U K$. less, in most of the studies, the wind effects are ignored, though freak waves are often observed being accompanied with strong winds in reality (e.g. [17]). The presence of the winds may dramatically influence the property of freak waves generated by other mechanisms. This has been experimentally confirmed by Giovanangeli et al. [2], Touboul et al. [20] and Kharif et al. [6], who concluded that the winds shift the focusing point and increase the wave amplitude for specific two-dimensional (2-D) freak waves. Therefore, ignoring wind effects may underestimate the maximum wave height and/or lead to incorrect prediction of the focusing point/time.

So far, studies relating to wind effects on freak waves mainly focus on three aspects. The first one is the feature of air flow structure above the free surface $[6,12,20,25]$. The second one is the mechanism of energy/momentum exchanging between the wind and the freak waves [6] or the feature of the temporal-spatial distribution of the free surface pressure due to wind $[25,27]$. The third aspect is about how the feature of the freak wave changes under the action of wind. The outcome of studies addressing the last aspect benefits the accurate prediction of the focusing time/point and maximum wave height of a freak wave under winds. In this aspect, Touboul et al. [20, 21] and Kharif et al. [6] studied the elevation amplification of 2-D freak waves, which are generated by spatiotemporal focusing or modulation instability, under different winds in deep water; Ma et al. [12] and Yan et al. [25] carried out a similar study but focused on 2-D shallow-water freak waves. The studies published related to this aspect pay more attention to the significance of the wind action on a specific freak wave in terms of wind speeds. Our previous investigation has shown that for a specific wind speed, a freak wave [25] seems to be much easier to be deformed than a solitary wave [24]. This implies that the significance of the wind action may also strongly depend on the freak wave itself, specifically on the parameters which govern the freak waves (e.g. focusing time/point and frequency range). However, a systematic investigation addressing this has not been found in the public domain.

In this paper, such investigations will be carried out only on 2-D freak waves. Although $2 \mathrm{D}$ cases are very rare in reality, investigations on 2D cases can shed some light on main issues and the corresponding results may be used for useful reference 
for 3D studies. Different freak waves generated using different focusing point/time and frequency ranges will be considered in the paper. The significance of the wind action on different freak waves will be examined and discussed.

\section{MATHEMATICAL MODEL AND NUMERICAL APPROACH}

In this paper, the computational domain is chosen as a rectangular tank. The Freak wave is generated in the tank by a piston-like wavemaker. The wavemaker is mounted at the left end and a damping zone with a Sommerfeld condition (see [10] for details) may be applied at the right end in order to suppress the reflection. A Cartesian coordinate system is used with the oxy plane on the mean free surface and with the $z$-axis being positive upwards. The origin of the coordinate system is located at the left end of the tank. Winds with speed of $U_{w}$ may be introduced along $x$-direction. A constant $x$-direction current may be added to model the effect of the wind-driven current. A fully nonlinear potential theory is used to describe the problem, in which the total velocity potential $(\Phi)$ is expressed by

$$
\Phi=\phi+x U_{c}
$$

where $U_{c}$ is the current speed and $\phi$ is the rest of the velocity potential apart from $x U_{c}$. In the fluid domain, the velocity potential satisfies the Laplace's equation,

$$
\nabla^{2} \phi=0
$$

On the wavemaker, the boundary condition reads

$$
\frac{\partial \phi}{\partial n}=\vec{n} \cdot \vec{U}(t)
$$

where $\vec{U}(t)$ and $\vec{n}$ are the oscillating velocity and the outward unit normal vector of the rigid boundaries, respectively. On the free surface $z=\zeta(x, y, t), \phi$ satisfies the kinematic and dynamic conditions in the following Lagrangian form,

$$
\begin{gathered}
\frac{D x}{D t}=\frac{\partial \phi}{\partial x}+U_{c}, \frac{D y}{D t}=\frac{\partial \phi}{\partial y}, \frac{D z}{D t}=\frac{\partial \phi}{\partial z} \\
\frac{D \phi}{D t}=-g z+\frac{1}{2}|\nabla \phi|^{2}+p_{s f}-\frac{U_{c}^{2}}{2}
\end{gathered}
$$

in which $D / D t$ is the substantial (or total time) derivative following fluid particles and $g$ is the gravitational acceleration. $p_{s f}$ is the wind-excited free-surface pressure, which may be estimated by using the Jeffreys' sheltering mechanism [4, 5], Miles' shearing mechanism [15, 16], Philips' model [18], Benjamin's model [1] for normal waves. However these models without modification may not be suitable for freak waves due to the strong nonlinearity involved in freak waves. Alternatively, one has employed the modified Jeffreys' theory proposed by Touboul et al. [20, 21] and Kharif et al. [6] and an improved model recently suggested by the authors of this paper [27]. According to our previous investigations [27], the improved model is chosen here. In this model, $p_{s f}$ is calculated by using,

$$
p_{s f}=\rho_{a}\left(U_{w}-c_{g}-U_{c}\right)^{2}\left(C_{a} k_{c} \zeta+C_{b} \frac{\partial \zeta}{\partial x}\right)
$$

where $\rho_{a}$ is the air density; $C_{g}$ is the group velocity of the freak wave. $C_{a}$ and $C_{b}$ are coefficients. Based on numerical tests [27], they are given in terms of $U^{\prime}=\left(U_{w}-C_{g}-U_{c}\right) / \sqrt{g d}$ by,

$$
\begin{gathered}
C_{a}=0.1344 U^{13}-0.9394 U^{12}+1.9654 U^{\prime}-1.3881 \\
C_{b}=-0.0170 U^{13}+0.1369 U^{\prime 2}-0.3786 U^{\prime}+0.5204
\end{gathered}
$$

Apart from the wind-excited pressure, another effect considered is the wind-driven current, i.e. $U_{c}$ in Eq. (1). Similar to [6], [21] and [27], a constant current is introduced and the magnitude of the current is specified as $0.5 \%$ wind speed. More extensive studies on the effects of current with different values will be published elsewhere in future.

The problem formed by Eqs. (1)-(5) is solved by the arbitrary Lagrangian-Eulerian finite element (QALE-FEM) method, which is developed by the authors of this paper and been proven to be the fastest method at the same accuracy level for fully nonlinear waves $[11,13,23,26]$, using a time-marching procedure. At each time step, the boundary value problem for the velocity potential $\phi$ is solved by the FEM. The details about the FEM formulation have been described in our previous publications [10] and will not be repeated here. The main difference between the QALE-FEM method and the conventional FEM method [10] mainly includes two aspects when they are applied to modeling wave problems without structures. One is that the computational mesh is moving in the QALE-FEM method, instead of being regenerated, at every time step during the calculation. To do so, a novel methodology has been suggested to control the motion of the nodes, in which interior nodes and nodes on the free surface (freesurface nodes) are separately considered. Different methods are employed to move different groups of nodes. The other aspect of the difference between the QALE-FEM and conventional FEM methods is the calculation of the fluid velocity on the free surface. The technique developed in the former is suitable for computing the velocity when waves become very steep or even overturning. More details of these techniques can be found in $[11,13,23,26]$. It is noted that the formulation here does not take into account the viscosity. It is acceptable for cases without post-breaking considered in this 
paper. If the cases with post-breaking are of main concern, other formulations based on the Navier-Stokes equations should be employed, such as in [14, 19, 28, 29].

\section{NUMERICAL RESULTS AND DISCUSSIONS}

As indicated above, the significance of the wind action not only depends on the wind speeds, but also depends on the wave parameters. These issues have not been systematically investigated so far to the best of our knowledge. In this section, three wave-related parameters, i.e. focusing position, focusing time and initial frequency range, are considered. For convenience, the parameters with a length scale are nondimensionalised by the water depth $d$; the time and frequency $(\omega)$, by $t \rightarrow \tau \sqrt{d / g}, \omega \rightarrow \omega \sqrt{g / d}$ where $\tau$ is the dimensionless time and $g$ is the gravitational acceleration. Because the model formed by Eqs. (1)-(5) is three-dimensional (3-D). To use it for modeling 2-D cases, the width of the tank is taken the same as the water depth and all parameters are not allowed to vary along $y$-direction, making it a $y$-independent 3-D problem. The convergence property of the method has been demonstrated in our previous publications [9, 11, 23, 25-27]. In order to focus on the discussions and analysis of the results concerned here, the investigations on the convergence will not be shown, though these have been carried out for all cases presented in the paper.

\section{Freak Wave Generation}

The 2-D freak waves are generated by the spatio-temporal focusing mechanism, i.e. a sum of a number of sin (cosine) wave components, using a piston-type wavemaker. The displacement of the wavemaker is given by

$$
S(\tau)=\sum_{n=1}^{N} \frac{a_{n}}{F_{n}} \cos \left(\omega_{n} \tau+\varepsilon_{n}\right)
$$

where $N$ is the total number of components and

$$
F_{n}=\frac{2\left[\cosh \left(2 k_{n}\right)-1\right]}{\sinh \left(2 k_{n}\right)+2 k_{n}}
$$

is the transfer function of the wavemaker [9]. $k_{n}$ and $\omega_{n}$ are the wave number and frequency of the $n$-th component, respectively. They are related to each other by $\omega_{n}{ }^{2}=k_{n} \tanh \left(k_{n}\right)$. The frequency of the wave components are equally spaced over the range $\left[\omega_{\min }, \omega_{\max }\right] . \quad \varepsilon_{n}$ is the phase of the $n$-th component and is chosen to be $k_{n} x_{f}-\omega_{n} \tau_{f}$ with $x_{f}$ and $\tau_{f}$ being the linear focusing point and the focusing time. $a_{n}$ is the individual amplitude of $n$-th component, which is taken as the same for all components in this paper to simplify the relationship between the target amplitude $\left(A_{t}\right)$ of the freak wave and the amplitudes of the components, leading to $a_{n}=A_{t} / N$.

It should be noted that $x_{f}$ and $\tau_{f}$, which is required to generate the freak waves, represents where and when the wave

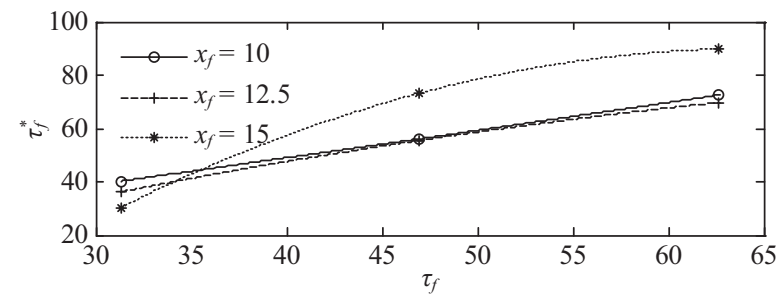

(a)

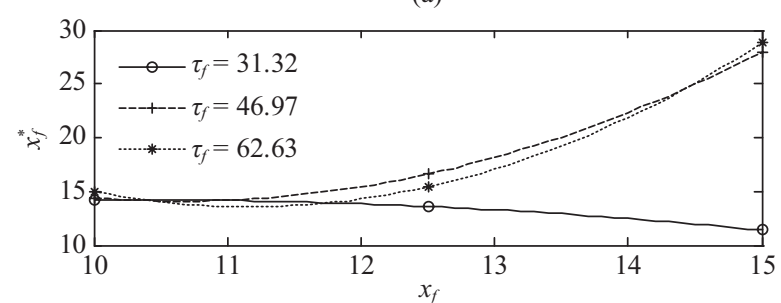

(b)

Fig. 1. (a) Focusing point $x_{f}{ }^{*}$ and (b) focusing time $\tau_{f}^{*}$ in the cases with different $x_{f}$ and $\tau_{f}\left(\omega_{\min }=0.5, \omega_{\max }=1.4, N=32, a_{n}=0.008, U_{w}=0\right)$.

components become phase coherent in the linear theory [9]; i.e., according to the linear theory, the wave elevation or wave height reaches its maximum value at the location $x=x_{f}$ and the time $\tau=\tau_{f}$. However, due to the nonlinearity and wind effects, the phase coherent may never happen [7] or the maximum wave elevation occurs at a position and time different from $x_{f}$ and $\tau_{f}[9,23]$. Therefore, it would be more sensible to use the location $\left(x_{f}^{*}\right)$ and time $\left(\tau_{f}^{*}\right)$ corresponding to the highest crest to indicate the occurrence of freak waves [23]. $x_{f}{ }^{*}$ and $\tau_{f}^{*}$ are generally different from the linear values of $x_{f}$ and $\tau_{f}$. For clarity, $x_{f}{ }^{*}$ and $\tau_{f}{ }^{*}$ are referred to as the focusing point and time, $x_{f}$ and $\tau_{f}$, are referred to as the coherent point and time, respectively. Preliminary studies on $x_{f}{ }^{*}$ and $\tau_{f}^{*}$ in the cases with different $x_{f}$ and $\tau_{f}$ without winds are carried out in order to generate suitable freak waves for further investigations with winds. Fig. 1 shows an example of the focusing point/time in the cases with different $x_{f}$ and $\tau_{f}$, in which, $\omega_{\min }=0.5, \omega_{\max }=1.4, N=32, a_{n}=0.008, U_{w}=0$. This figure clearly confirms that $x_{f}{ }^{*}$ and $\tau_{f}^{*}$ are different from $x_{f}$ and $\tau_{f}$ for all cases. It is also observed that $\tau_{f}{ }^{*}$ increases as $\tau_{f}$ increases in the cases with different $x_{f}$ (Fig. 1 (a)). Nevertheless, $x_{f}{ }^{*}$ may increase (e.g. $\tau_{f}=46.97$ and $\tau_{f}=62.63$ ) or decrease (e.g. $\tau_{f}=31.32$ ) with the increase of $x_{f}$. For convenience, the focusing point and focusing time in the cases without wind are denoted by $x_{f, 0}^{*}$ and $\tau_{f, 0}^{*}$, respectively, hereafter.

It should also be noted that due to the occurrence of the wave overturning, the simulation stops before the postbreaking occurs, for which the potential theory may not be applicable as indicated above. Nevertheless, the breaking event causes the wave elevation downstream to become smaller due to the energy dissipation, as demonstrated in [25]. Therefore, the highest elevation before the overturning occurs could be considered as the highest elevation appears in the whole domain, i.e. the focusing point $\left(x_{f}{ }^{*}\right)$, and the corresponding time is the focusing time $\tau_{f}{ }^{*}$. 


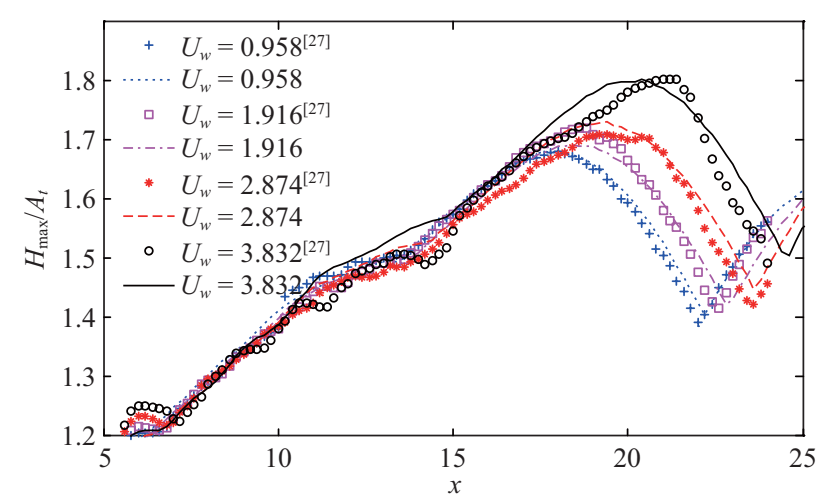

Fig. 2. Comparison of maximum wave height recorded at different positions $\left(\omega_{\min }=0.5, \omega_{\max }=1.4, N=32, a_{n}=0.008, x_{f}=12.5, \tau_{f}=46.97\right.$; the superscript in the legend represents the results from QALE-FEM/ StarCD approach in reference [27]).

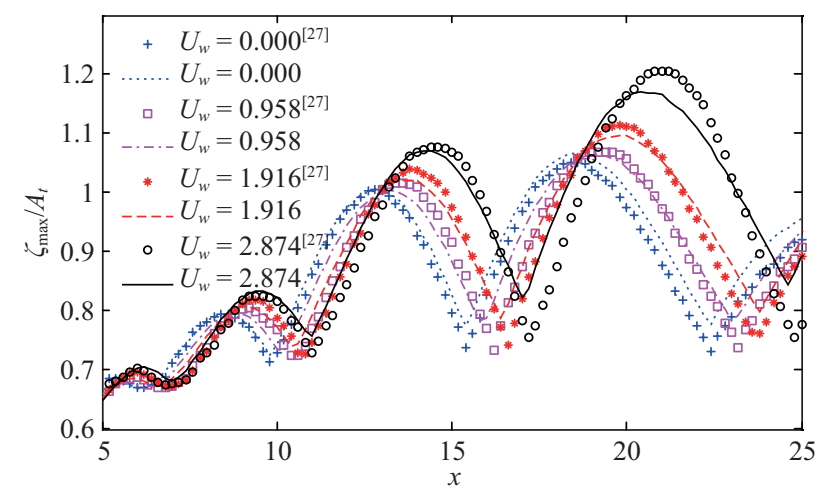

Fig. 3. Comparison of maximum elevation recorded at different positions $\left(\omega_{\min }=0.8, \omega_{\max }=1.6, N=32, a_{n}=0.00575, x_{f}=15, \tau_{f}=46.97\right.$; the superscript in the legend represents the results from QALE-FEM/ StarCD approach in reference [27]).

\section{Numerical Validation and Typical Feature of Freak Waves under Winds}

As indicated in Section II, a wind-excited pressure $p_{s f}$ is introduced in the dynamic free surface condition to model the wind effect. Though the shear stress is not considered, the justification of the numerical strategy, i.e. employing a winddriven pressure on the free surface condition of the FNPT model, has been numerically confirmed by Touboul et al. [20, 21] and Kharif et al. [6] for simulating wind effects on $2 \mathrm{D}$ freak waves. Apart from this, comparisons between the QALEFEM adopting the improved pressure model, i.e. Eqs. (6)-(8) and a numerical approach namely QALE-FEM/StarCD [25], which fully couples the air flow and wave motions and therefore considers the sheer stress on the free surface, have also suggested that ignoring the sheer stress may be acceptable for simulating wind effects on 2D freak waves whose time scale is normally much shorter than the wind-wave generation procedure [27]. More comparisons are given here to demonstrate the validity of the present model.

Fig. 2 illustrates a typical spatial variation of maximum wave height $\left(H_{\max }\right)$ estimated by using two consecutive crests

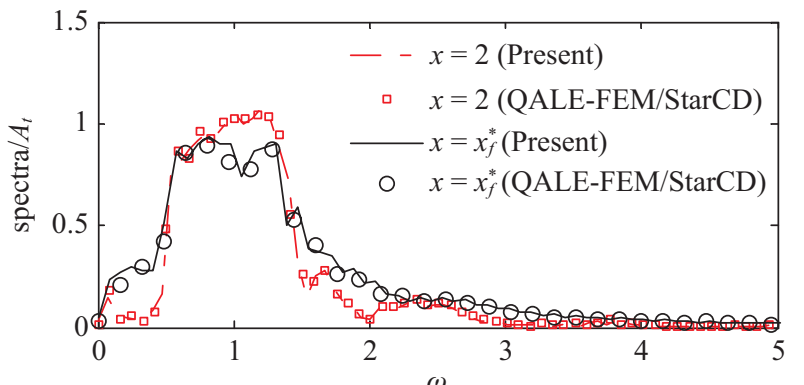

(a)

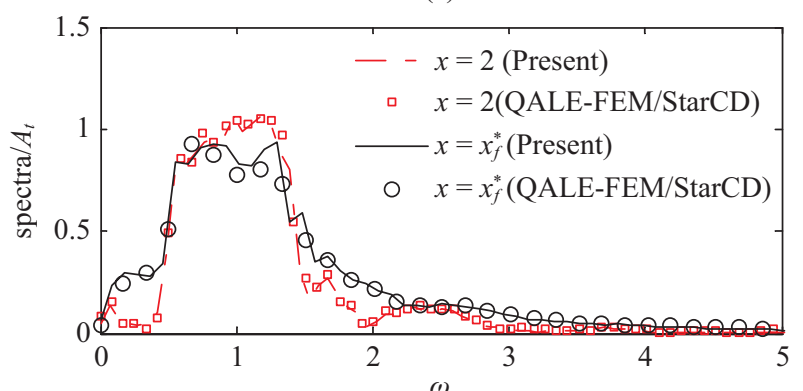

(b)

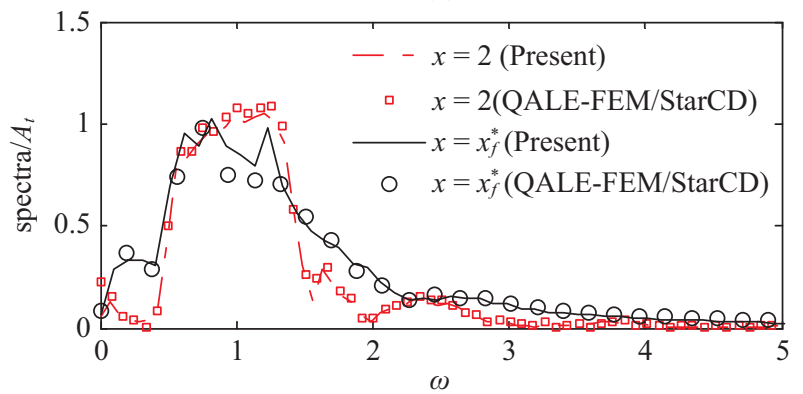

(c)

Fig. 4. Spatial variation of spectra for (a) $U_{w}=0$, (b) $U_{w}=1.915$ and (c) $U_{w}=3.832\left(\omega_{\min }=0.5, \omega_{\max }=1.4, N=32, a_{n}=0.008, x_{f}=12.5, \tau_{f}=\right.$ 46.97).

and troughes in wave histories in the cases with different wind speeds. In the cases, $\omega_{\min }=0.5, \omega_{\max }=1.4, N=32, a_{n}=$ $0.008, x_{f}=12.5, \tau_{f}=46.97$. Winds with speed ranging from 0 to 3.832 are posed in the direction of the wave propagation. This figure identifies the amplification of wave height and the shift of the focusing point due to the winds, consistent with the experimental results [6]. For the purpose of comparison, the corresponding results from the QALE-FEM/StarCD [27] are also plotted together. As shown, the results from two different numerical methods are close. Comparisons are also made for other waves with different wave heights and spectra. A similar agreement has been achieved. Only one more example is given in Fig. 3 for demonstration.

Apart from the spatial variation of the wave elevation/wave height, the spatial variation of spectra is also analyzed, which is obtained by performing Fourier analysis on time histories at given points. Some results for the cases shown in Fig. 2 are plotted in Fig. 4. For convenience, the spectra is divided by $A_{t}$. From this figure, it is found that the spectrum of the freak wave changes during the propagation and the energy seems to 


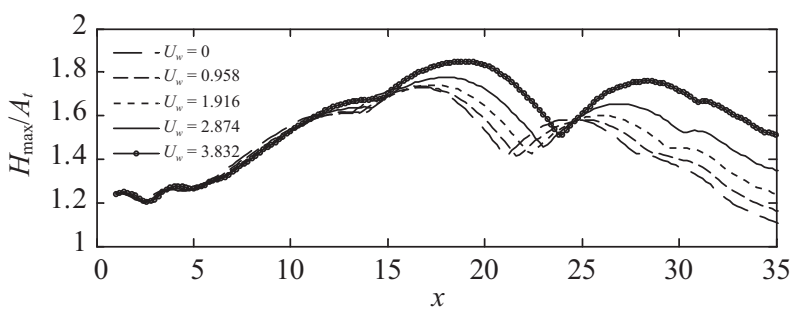

(a)

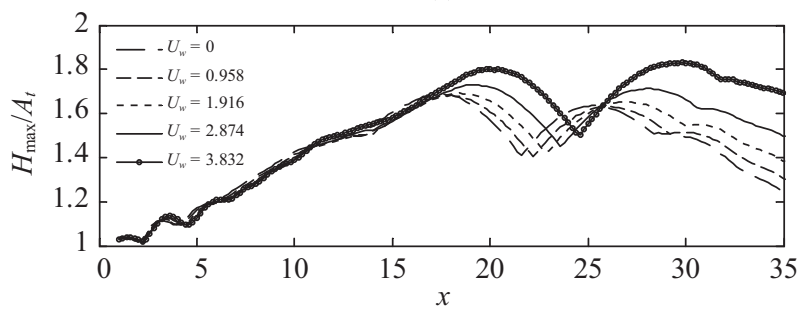

(b)

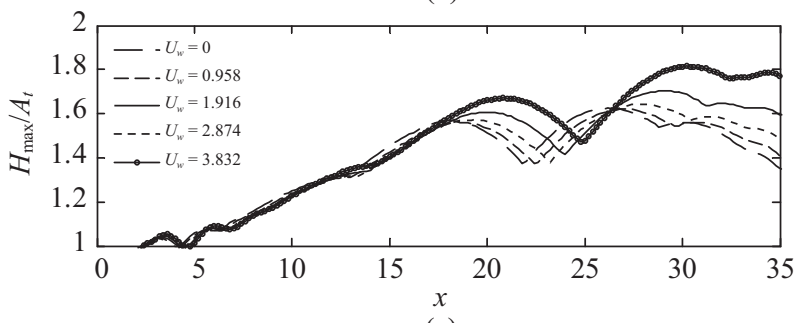

(c)

Fig. 5. Maximum wave height recorded at different positions subject to different wind speeds with (a) $x_{f}=10\left(x_{f, 0}^{*}=14.4\right)$; (b) $x_{f}=12.5$ $\left(x_{f, 0}^{*}=16.8\right)$ and (c) $x_{f}=15\left(x_{f, 0}^{*}=28.0\right)\left(\omega_{\min }=0.5, \omega_{\max }=1.4, N=\right.$ $\left.32, a_{n}=0.008, \tau_{f}=46.97\right)$.

be transferred from the fundamental harmonics, i.e. frequency ranging between 0.5 and 1.4 , to both lower harmonics and higher harmonics. Again, the agreements between the present results and those from the QALE-FEM/StarCD approach are satisfactory, for all wind speeds considered.

\section{Effects of $x_{f, 0}^{*}$}

In this sub-section, the effect of $x_{f, 0}^{*}$ on the significance of the wind action on freak waves is investigated, followed by the effect of $\tau_{f, 0}^{*}$ in the next sub-section.

To obtain freak waves with different $x_{f, 0}^{*}$, we assign different $x_{f}$ for specifying the wavemaker motion. In the investigation, $\omega_{\min }=0.5, \omega_{\max }=1.4, N=32, a_{n}=0.008, \tau_{f}=46.97$, $N=32$. The linear group velocity $\left(U_{g}\right)$ and the wave number $\left(k_{c}\right)$ corresponding to the wave component with the central frequency are 0.5972 and 1.12. Different values of $x_{f}$, ranging from 10 to 15 are used. The corresponding $x_{f, 0}^{*}$ ranges from 14.4 to 28 as shown in Fig. 1(a). Different wind speeds varying from 0 to 3.832 are used. The maximum wave height $\left(H_{\max }\right)$ is examined. The results for the cases with different wind speeds are plotted in Fig. 5.

From Fig. 5, it is observed that the location where the maximum wave height appears, i.e. the focusing point, is shifted significantly further downstream by the wind in all the cases. It is also found that the highest wave height seems to be

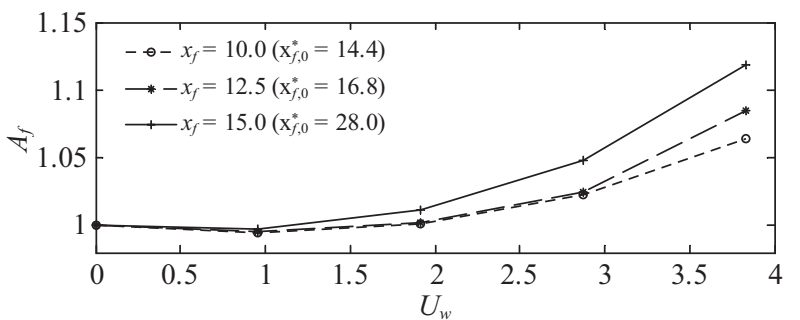

(a)

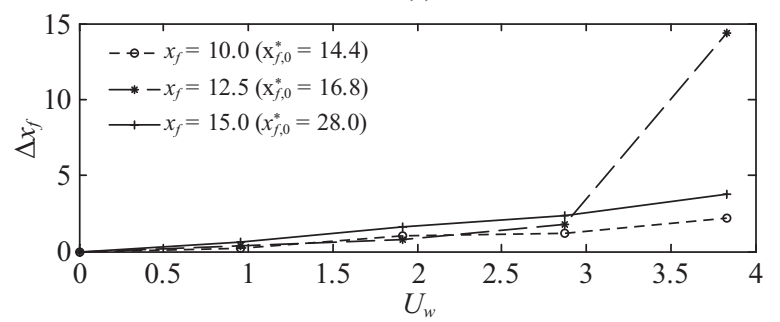

(b)

Fig. 6. (a) Amplification factor and (b) shift distance as a function of wind speeds in the cases with different $x_{f, 0}^{*}\left(\omega_{\min }=0.5, \omega_{\max }=1.4\right.$, $N=32, a_{n}=0.008, \tau_{f}=46.97$ )

not affected by the wind with small speeds, i.e. $U_{w}=0.958$, whilst, for stronger winds, the highest wave height are increased dramatically. These observations are largely consistent with the experiments by Kharif et al. [6], which also concluded that the wind may pose effects on the formation of freak waves in two ways, i.e. the shift of the focusing point and the amplification of the wave height. In order to quantitatively examine the significance of wind action, two parameters, i.e. the shift distance of the focusing point $\left(\Delta x_{f}\right)$ and amplification factor $\left(A_{f}\right)$, are defined. The former reflects how the wind shifts the focusing point and its value is given by

$$
\Delta x_{f}=x_{f}^{*}-x_{f, 0}^{*}
$$

The latter is calculated using

$$
A_{f}=H_{\max } / H_{\max }^{0}
$$

where $H_{\text {max }}$ and $H^{0}{ }_{\text {max }}$ are the maximum wave height observed in the case with wind and that without wind, respectively. The corresponding shift distance of the focusing point $\left(\Delta x_{f}\right)$ and amplification factor $\left(A_{f}\right)$ in the cases shown in Fig. 5 are plotted in Fig. 6.

From Fig. 6(a), it is observed that the amplification factor $A_{f}$, and so the nonlinearity, increases, i.e. the significance of the wind action becomes stronger, for all wind speeds considered as $x_{f, 0}^{*}$ increases. In addition, a stronger wind causes a larger wind-driven current. According to previous studies, both the wind-driven current (Kharif et al. [6]; Yan et al. [27]) and the nonlinearity [9] shift the focusing point further downstream in cases without involving wave breaking. This is confirmed by the results shown in Fig. 6(b), which clearly 


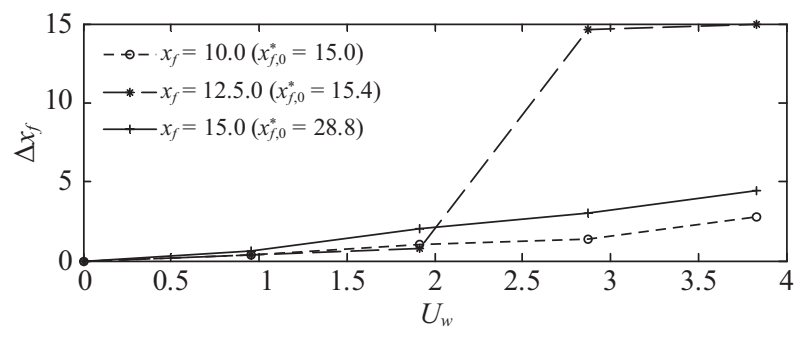

Fig. 7. Shift distance as a function of wind speeds in the cases with different $x_{f, 0}^{*}\left(\omega_{\min }=0.5, \omega_{\max }=1.4, N=32, a_{n}=0.008, \tau_{f}=62.63\right)$.

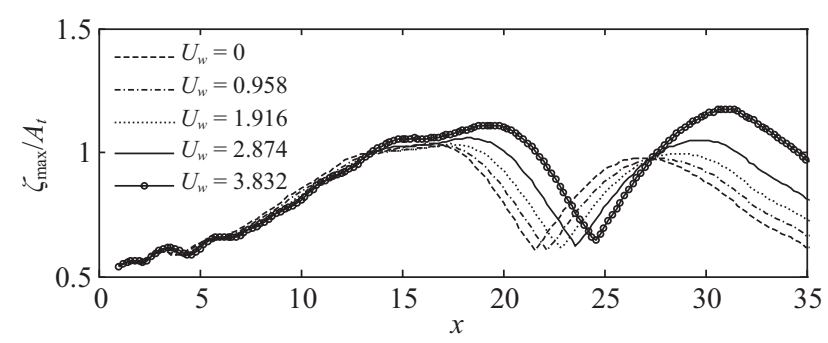

Fig. 8. Maximum wave elevation recorded at different positions with different wind speeds $\left(\omega_{\min }=0.5, \omega_{\max }=1.4, N=32, a_{n}=0.008, \tau_{f}=\right.$ 46.97, $x_{f}=12.5$ ).

shows that the shift distance $\left(\Delta x_{f}\right)$ increases with the increase of the wind speed for all $x_{f, 0}^{*}$. From Fig. 6(b), it is also observed that for a specific wind speed less than 3 , the shift distance $\left(\Delta x_{f}\right)$ increases as the $x_{f, 0}^{*}$ increases. However, one may find that when $U_{w}=3.832$, the shift distance does not follow the trend, i.e., $\Delta x_{f}$ in the case with $x_{f, 0}^{*}=15.4$ is much larger than those with other $x_{f, 0}^{*}$. A similar phenomenon is also found in other cases, e.g. the one shown in Fig. 7, in which the shift distance for $x_{f, 0}^{*}=15.4$ significantly increases from about 0.8 to 14.6 when the wind speed increases from 1.916 to 2.874 . To explore the reason, the maximum elevations, which are used to identify the location where the freak wave occurs and to determine $x_{f}^{*}$, recorded at different positions under different winds in the case with $x_{f, 0}^{*}=15.4\left(x_{f}=\right.$ 12.5) shown in Fig. 6 are illustrated in Fig. 8. As can be seen from the figure, all curves have two crests, one near $x=17.5$ and the other one located further downstream. For the cases with the wind speed less than 3.832, the focusing points are located around $x=17.5$, i.e. the first crest of each curves; whereas, for the case with $U_{w}=3.832$, the second crest is higher than the first crest. This explains why $\Delta x_{f}$ becomes very large in Fig. 6 and Fig. 7.

Other than those shown in Fig. 6 and Fig. 7, the cases with different $x_{f, 0}^{*}$ are also tested, in which $\omega_{\min }=0.5, \omega_{\max }=1.4$, $N=32, a_{n}=0.008, \tau_{f}=31.32$ and $x_{f}$ varies from 10 to 15 . Using this configuration, $x_{f, 0}^{*}$ decreases from 14.2 to 11.1 as $x_{f}$ increases as can be seen in Fig. 9. Fig. 9 also shows that, for a large wind speed, e.g. $U_{w}=3.832$, the amplification factor increases as $x_{f, 0}^{*}$ increases while it change little for a small wind speed. This is consistent with the results shown in

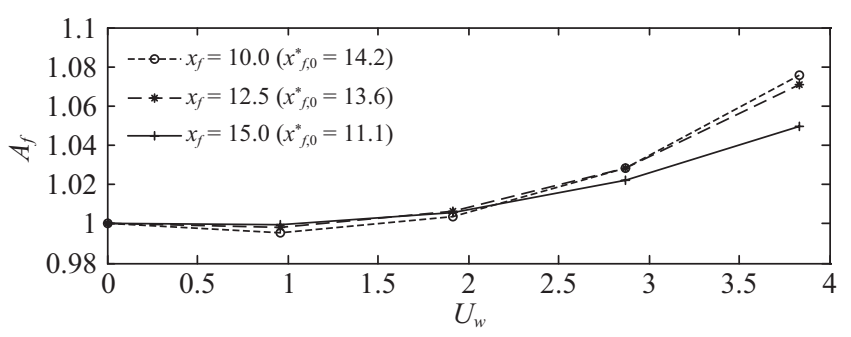

Fig. 9. Amplification factor as a function of wind speeds in the cases with different $x_{f, 0}^{*}\left(\omega_{\min }=0.5, \omega_{\max }=1.4, N=32, a_{n}=0.008, \tau_{f}=31.32\right)$.

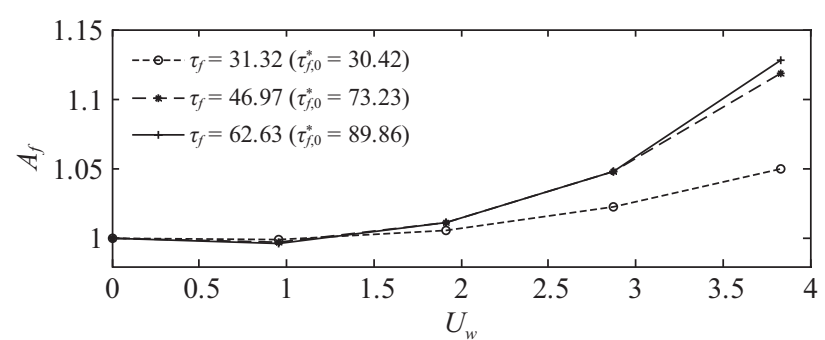

(a)

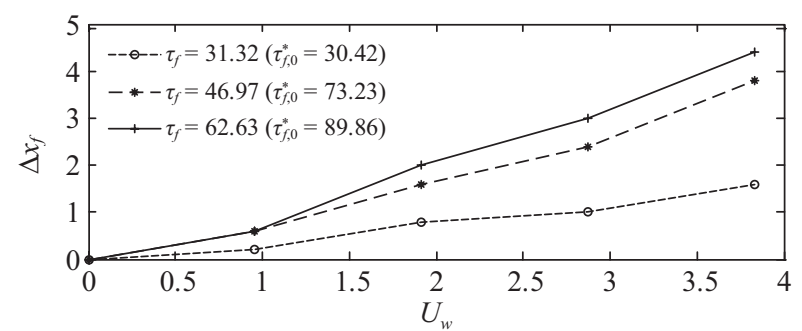

(b)

Fig. 10. (a) Amplification factor $A_{f}$ and (b) shift distance $\Delta x_{f}$, as a function of wind speeds in the cases with different $\tau_{f, \theta}^{*}\left(\omega_{\min }=0.5\right.$, $\omega_{\max }=1.4, N=32, a_{n}=0.008, x_{f}=15$ ).

Fig. 7. Nevertheless, if the focusing point is denoted by $x_{f}$ (the linear coherent point), Fig. 9 would lead to an opposite conclusion, i.e. the amplification factor decreases as $x_{f}$ decreases. This indicates that the trend of significance of winds on the wave height depends on the definition of the location where the freak wave occurs. The focusing point defined and used here lead to consistent trend in all the cases.

\section{Effects of $\tau_{f, 0}^{*}$}

The effect of $\tau_{f, 0}^{*}$ is investigated here. A typical variation of the amplification factor and shift distance against wind speeds in the cases with different $\tau_{f, 0}^{*}$ is shown in Fig. 10, in which $\omega_{\min }=0.5, \omega_{\max }=1.4, N=32, a_{n}=0.008 . x_{f}=15$ and $\tau_{f, 0}^{*}$ ranges from 30.42 to 89.86 (the corresponding $\tau_{f}$ is chosen between 31.32 and 61.63). This figure shows that both the amplification factor and the shift distance increases as $\tau_{f, 0}^{*}$ increases when wind speeds are sufficiently large.

Fig. 11 displays another example, in which $\omega_{\min }=0.5$, $\omega_{\max }=1.4, N=32, a_{n}=0.008, x_{f}=10.0$ and $\tau_{f, 0}^{*}$ varies from 40.29 to 72.57 (the corresponding $\tau_{f}$ ranges from 10 to 20). In this example, the amplification factor in the case with the 


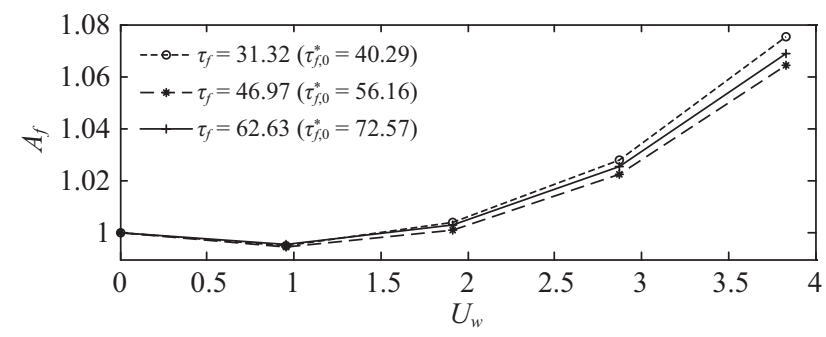

Fig. 11. Amplification factor $A_{f}$ as a function of wind speeds in the cases with different $\tau_{f, 0}^{*}\left(\omega_{\min }=0.5, \omega_{\max }=1.4, N=32, a_{n}=0.008, x_{f}=\right.$ 10.0).

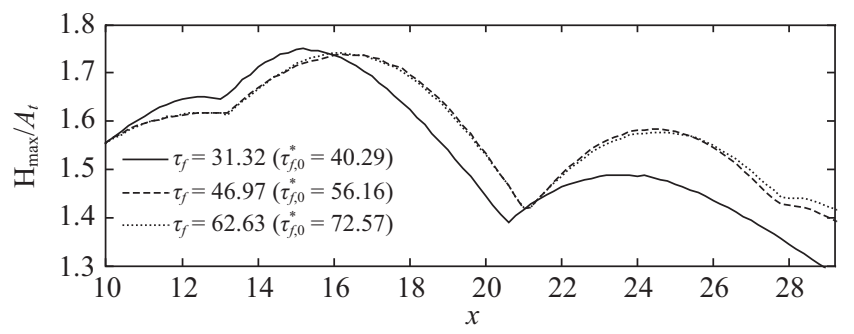

(a)

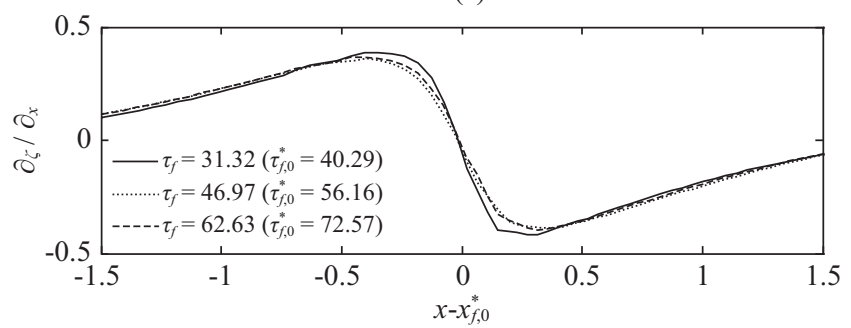

(b)

Fig. 12. (a) Maximum wave height recorded at different positions and (b) free surface slope recorded at the focusing time in the cases with different $\tau_{f, 0}^{*}\left(\omega_{\min }=0.5, \omega_{\max }=1.4, N=32, a_{n}=0.008, x_{f}=\right.$ 10.0, $\left.U_{w}=0\right)$.

smallest $\tau_{f, 0}^{*}(40.29)$ is larger than others. This may be explained through analyzing the feature of the freak wave without wind. For this purpose, the maximum wave height recorded at different positions and free surface profile recorded at the focusing time in the cases with different $\tau_{f, 0}{ }_{f 0}$ is plotted in Fig. 12 in which the wind is not considered. It is observed from Fig. 12(a) that the spatial distribution of maximum wave height in the case with smallest $\tau_{f, 0}^{*}$ is significantly different from others. The maximum wave height in this case is larger than others, though $\omega_{\min }, \omega_{\max }, N, a_{n}$ and $x_{f}$ in these cases are the same. In addition, the wave with the smallest $\tau_{f, 0}^{*}$ has higher wave slope and, therefore, is steeper than those with larger $\tau_{f, 0}^{*}$ (Fig. 12(b)). According to our previous studies [27], larger wave height or wave slope cause more significant asymmetry of pressure about the crest and, therefore, lead to more energy transfer from the wind to the freak wave. This may be one reason causing the amplification factor in the case with smallest $\tau_{f, 0}^{*}$ to be larger than others.

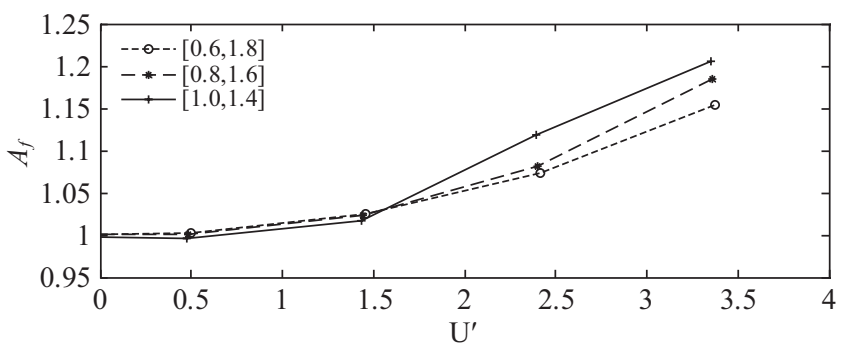

(a)

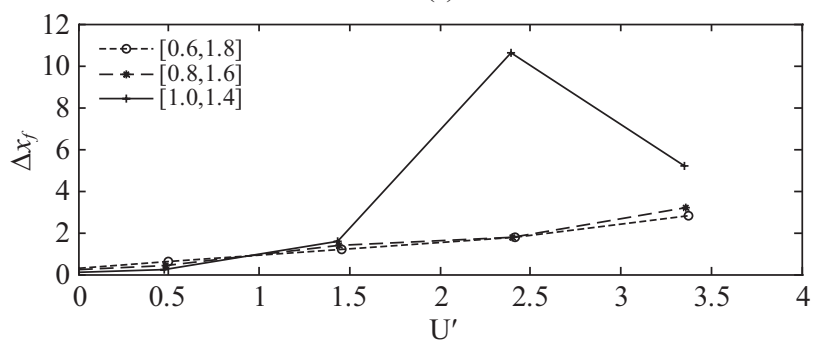

(b)

Fig. 13. (a) Amplification factor $A_{f}$ and (b) shift distance $\Delta x_{f}$, as a function of wind speeds in case with different frequency ranges $(N=$ 32, $x_{f}=15, \tau_{f}=46.97, K_{c} A_{t}=0.289$ ).

\section{Effects of Frequency Range}

Another factor which may affect the significance of wind actions on freak waves is the frequency range set when generating the freak wave. To shed some light on this, the cases with different frequency ranges are carried out. For all these cases, $K_{c} A_{t}$ are assigned to be a constant value, i.e. 0.289. $N=$ $32, \tau_{f}=46.97$ and $x_{f}=15$ are used in this investigations.

In the first cases considered here, the central frequency is fixed to be 1.2. Different range of frequency is chosen, i.e. $[0.6,1.8],[0.8,1.6]$ and $[1,1.4]$. The group velocities are $0.4595,0.4725$ and 0.4824 , respectively. Fig. 13 shows the shift distance and amplification factor as a function of wind speeds in the cases with different frequency ranges. For convenience, the horizontal axis uses $U^{\prime}=U_{w}-C_{g}-U_{c}$, because the group velocities are different for different cases.

From Fig. 13(a), it is found that the frequency range slightly influences the amplification factor when the wind speed is relatively smaller. However, when the wind speed is larger, the amplification factor increases as the width of the frequency range decreases. It is also observed from Fig. 13(b) that the change in the frequency range does not lead to a significant focusing point shift, except for the case with frequency range of $[1.0,1.4]$. In this case, the shift distance reaches a maximum at $U_{w}=2.874\left(U^{\prime} \approx 2.4\right)$ but fall back at $U_{w}=3.832\left(U^{\prime} \approx 3.4\right)$. The main reason may be similar to that shown in Fig. 6(b) and Fig. 7. To confirm this, the maximum wave elevation recorded at different positions in the case with the frequency range of $[1.0,1.4]$ is plotted in Fig. 14. This figure shows that when $U_{w}=2.874$, the highest elevation occurs at the last crest $(x \approx 35)$, whereas for $U_{w}<1.5$, the highest elevation occurs at the third crest around $x=25$. One 


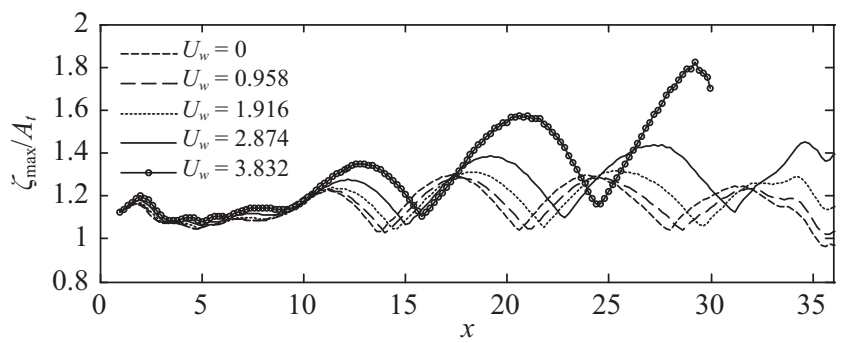

Fig. 14. Maximum elevation recorded at different positions in the cases with different wind speeds $\left(\omega_{\min }=1.0, \omega_{\max }=1.4, N=32, K_{c} A_{t}=\right.$ $\left.0.289, \tau_{f}=46.97, x_{f}=15\right)$.

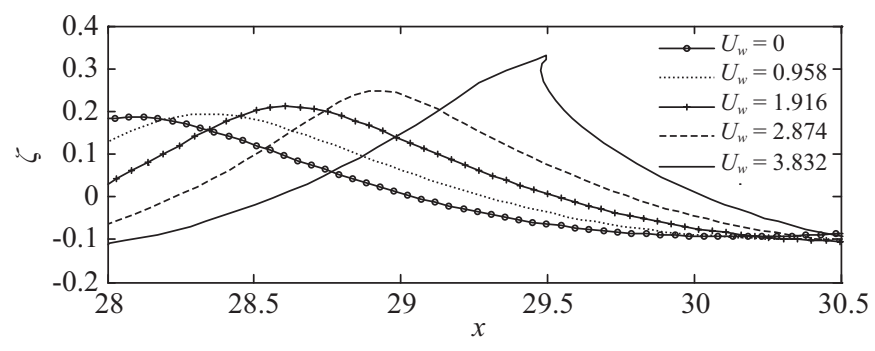

Fig. 15. Free surface profile at $\tau \approx 67.75$ in the cases with different wind speeds $\left(\omega_{\min }=1.0, \omega_{\max }=1.4, N=32, K_{c} A_{t}=0.289, \tau_{f}=46.97, x_{f}=\right.$ 15).

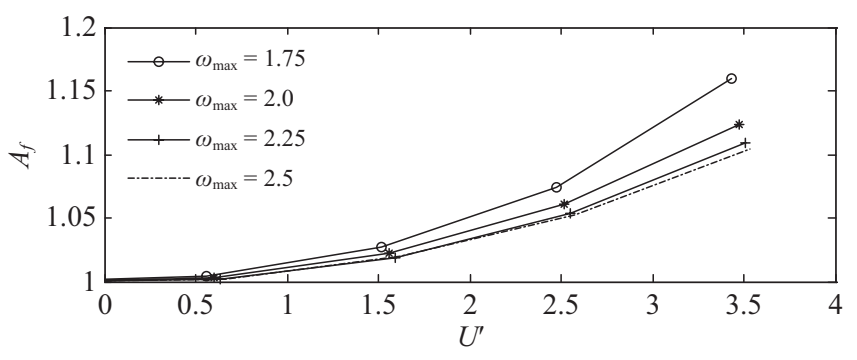

Fig. 16. Amplification factor $A_{f}$ as a function of wind speeds in the cases with different maximum frequencies $\left(N=32, x_{f}=15, \tau_{f}=46.97\right.$, $\left.K_{c} A_{t}=0.289, \omega_{\min }=1.0\right)$.

may also observe from Fig. 13(b) that the shift distance for the case with $U_{w}=3.832$ is smaller than that with $U_{w}=2.874$. This is due to the occurrence of a wave breaking in the former case as shown in Fig. 15, which illustrates the free surface profiles at a time step when an overturning jet appears near $x \approx$ 29.5 in the case with $U_{w}=3.832$. Such breaking event shifts the focusing points upstream as discussed in [23, 25].

In the second case considered here, the minimum frequency is fixed at $\omega_{\min }=1.0$, while different maximum frequencies, ranging from 1.4 to 3.0, are employed to generate the freak wave. Similar to those shown in Fig. $14, K_{c} A_{t}=0.289$ is used to give the wave amplitude. The amplification factors as a function of wind speeds in the cases with different maximum frequencies are shown in Fig. 16. From this figure, it is found that the amplification factor increases with the decrease of maximum frequency. This implies that for a given steepness, the wind causes a more significant effect on amplifying the wave height of a 2D freak wave with smaller frequency range, in line with what has been seen in Fig. 13.

\section{CONCLUSION}

This paper presents numerical investigations on how the significance of wind actions differs when varying the wave parameters. Our attention mainly focuses on the variation of the focusing point $\left(x_{f, 0}^{*}\right)$, focusing time $\left(\tau_{f, 0}^{*}\right)$ and frequency range. The results indicate that the significance of the wind actions on freak waves not only relies on the wind speed but also strongly depends on the parameters of the freak waves. For the same freak wave, stronger winds normally lead to larger wave elevations and shift the focusing point further downstream except the cases involved wave breaking, in which they shift the focusing point further upstream. For specific wind speed and wave steepness, the wind effects on amplifying the wave height is more significant for the case with larger focusing time/point, but narrower frequency range. The results obtained here may contribute to development of methods for predicting freak waves and for analyzing the local properties of the waves in winds. The knowledge also benefits the proper set up of experiments studying wind effects on freak waves.

\section{ACKNOWLEDGMENTS}

This work is sponsored by Leverhulme Trust, UK, for which the authors are most grateful.

\section{REFERENCES}

1. Benjamin, T. B., "Shearing flow over a wavy boundary," Journal of Fluid Mechanics, Vol. 6, pp. 161-205 (1959).

2. Giovanangeli, J. P., Kharif, C., and Pelinovsky, E., "Experimental study of the wind effect on the focusing of transient wave groups," Rogue Wave 2004, Brest, France, 2004 from http://www.ifremer.fr/web-com/stw2004/ rw/fullpapers/giovanangeli.pdf.

3. Haver, S., "A possible freak wave event measured at the Draupner Jacket January 1 1995," Rogue waves 2004, Brest, France, 2004 from http:// www.ifremer.fr/web-com/stw2004/rw/fullpapers/walk on haver.pdf.

4. Jeffreys, H., "On the formation of water waves by wind," Proceedings of the Royal Society of London, Series A, Vol. 107, pp. 189-206 (1925).

5. Jeffreys, H., "On the formation of water waves by wind (second paper)," Proceedings of the Royal Society of London, Series A, Vol. 110, pp. 241247 (1926).

6. Kharif, C., Giovanangeli, J. P., Touboul, J., Grare, L., and Pelnovsky, E., "Influence of wind on extreme wave events: Experimental and numerical approaches," Journal of Fluid Mechanics, Vol. 594, pp. 209-247 (2008).

7. Kharif, C. and Pelinovsky, E., "Physical mechanisms of the rogue wave phenomenon," European Journal of Mechanics - B/Fluids, Vol. 22, pp. 603-634 (2003).

8. Lawton, G., "Monsters of the deep (The perfect wave)," New Scientist, Vol. 170, pp. 28-33 (2001).

9. Ma, Q. W., "Numerical generation of freak waves using MLPG_R and QALE-FEM methods," Computer Modeling in Engineering \& Sciences (CMES), Vol. 18, pp. 223-234 (2007).

10. Ma, Q. W., Wu, G. X., and Eatock Taylor, R., "Finite element simulation of fully non-linear interaction between vertical cylinders and steep waves. Part 2: Numerical results and validation," International Journal for $\mathrm{Nu}$ - 
merical Methods in Fluids, Vol. 36, pp. 287-308 (2001).

11. Ma, Q. W. and Yan, S., "Quasi ALE finite element method for nonlinear water waves," Journal of Computational Physics, Vol. 212, pp. 52-72 (2006).

12. Ma, Q. W. and Yan, S., "Preliminary simulation on wind effects on 3D freak waves," Rogue Wave 2008, Brest, France, 2008 from http://www. ifremer.fr/web-com/stw2008/rw/abstracts/MaYan_2008.pdf.

13. Ma, Q. W. and Yan, S., "QALE-FEM for numerical modelling of nonlinear interaction between 3D moored floating bodies and steep waves," International Journal for Numerical Methods in Engineering, Vol. 78 , pp. 713-756 (2009).

14. Ma, Q. W. and Zhou, J. T., "MLPG_R method for numerical simulation of 2D breaking waves," Computer Modelling in Engineering \& Sciences(CMES), Vol. 43, No 3, pp. 277-303 (2009).

15. Miles, J. W., "Surface-wave generation revisited," Journal of Fluid Me chanics, Vol. 256, pp. 427-441 (1993).

16. Miles, J. W., "Surface-wave generation: a viscoelastic model," Journal of Fluid Mechanics, Vol. 322, pp. 131-145 (1996).

17. Mori, N., Liu, P. C., and Yasuda, T., "Analysis of freak wave measurements in the Sea of Japan," Ocean Engineering, Vol. 29, pp. 1399-1414 (2002).

18. Phillips, O. M., "On the generation of waves by turbulent wind," Journal of Fluid Mechanics, Vol. 2, pp. 417-445 (1957).

19. Qian, L., Causon, D. M., Mingham, C. G., and Ingram, D. M., "A freesurface capturing method for two fluid flows with moving bodies," Proceedings of the Royal Society of London, Series A, Vol. 462, pp. 21-42 (2006)

20. Touboul, J., Giovanangeli, J. P., Kharif, C., and Pelinovsky, E., "Freak waves under the action of wind: experiments and simulations," European
Journal of Mechanics - B/Fluids, Vol. 25, pp. 662-676 (2006).

21. Touboul, J. and Kharif, C., "On the interaction of wind and extreme gravity waves due to modulational instability," Physics of Fluids, Vol. 18, 108103 (2006).

22. Touboul, J., Pelinovsky, E., and Kharif, C., "Nonlinear focusing wave group on current," Journal of Korean Society of Coastal and Ocean Engineers, Vol. 19, pp. 222-227 (2007).

23. Yan, S. and Ma, Q. W., "Nonlinear simulation of 3-D freak waves using a fast numerical method," International Journal of Offshore and Polor Engineering, Vol. 19, pp. 168-175 (2009).

24. Yan, S. and Ma, Q. W., "Numerical simulation of wind effects on breaking solitary waves," Proceeding of 19th International Offshore and Polar Engineering Conference (ISOPE), Osaka, Vol. 3, pp. 480-487 (2009).

25. Yan, S. and Ma, Q. W., "Numerical simulation of interaction between wind and 2-D freak waves," European Journal of Mechanics - B/Fluids, Vol. 29, pp. 18-31 (2010).

26. Yan, S. and Ma, Q. W., "QALE-FEM for modelling 3D overturning waves," International Journal for Numerical Methods in Fluids, Vol. 63, pp. 743-768 (2010).

27. Yan, S. and Ma, Q. W., "Improved model for air pressure due to wind on 2D freak waves in shallow water," European Journal of Mechanics B/Fluids, Vol. 30, pp. 1-11 (2011).

28. Zhang, Y., Zou, Q., and Greaves, D. M., "Numerical simulation of two phase flow using the level set method with global mass correction," International Journal for Numerical Methods in Fluid, Vol. 63, pp. 651680 (2009).

29. Zhou, J. T. and Ma, Q. W., "MLPG Method based on Rankine source solution for modelling 3D Breaking Waves," Computer Modeling in Engineering \& Sciences (CMES), Vol. 56, No. 2, pp. 179-210 (2010). 